\title{
Revisión del género Macrolepiota Singer (Agaricales, Basidiomycota) en Chile
}

\author{
(Revision of the genus Macrolepiota Singer (Agaricales, Basidiomycota) \\ in Chile)
}

\begin{abstract}
Salazar-Vidal V. ${ }^{1 *}$, Dibán M.J. ${ }^{2}$ \& Ponce S. ${ }^{3}$
${ }^{1}$ Facultad de Ciencias Forestales, Universidad de Concepción, Victoria 631, Concepción, Chile ${ }^{2}$ Facultad de Ciencias, Universidad de Chile, Las Palmeras 3425, Nuñoa, Región Metropolitana ${ }^{3}$ Escuela de Medicina, Universidad de Valparaíso, Hontaneda 2653, Valparaíso.

*Autor para correspondencia: : vivianasalazar@udec.cl

RECIBIDO: 20 de Junio de 2017

APROBADO: 05 de Julio de 2017
\end{abstract}

DOI: 10.22370/bolmicol.2017.32.1.687

LOS AUTORES DECLARAN NO TENER CONFLICTO DE INTERESES

Palabras claves: Macrolepiota, Chlorophyllum, clave, taxonomía, morfología.

Key words: Macrolepiota, Chlorophyllum, key, taxonomy, morphology.

\section{RESUMEN}

El género Macrolepiota cuenta con 30 especies ampliamente distribuidas en el mundo, siendo éstas saprobiontes. En Chile se han documentado tres especies: $M$. procera, $M$. bonaerensis y $M$. rachodes, perteneciendo actualmente esta última al género Chlorophyllum. En esta revisión, se describen según la literatura los caracteres macromorfológicos y microscópicos de las tres especies mencionadas con anterioridad. Adicionalmente, se adaptó una clave dicotómica en base a la bibliografía para facilitar la determinación de las especies.

\section{ABSTRACT \\ The Macrolepiota genus include 30 species widely distributed around the world, being these saprobionts. In Chile, three species have been do-}

cumented: M. procera, M. bonaerensis y M. rachodes, at present the last specie currently belong to the genus Chlorophyllum. In this review, the macromorphological, and microscopic characteristics of the three species mentioned above are described according to the literature. In addition, a dichotomous key was adapted based on the bibliography to facilitate the determination of the species.

\section{INTRODUCCIÓN}

El género Macrolepiota Singer cuenta con 30 especies (Kirk et al., 2008), distribuidas ampliamente en América del Norte, Centroamérica, Brasil, Colombia, África, Europa, Japón, Oceanía (GBIF), China (Ge et al., 2010), Chile (Valenzuela, 2013) y Argentina (Wright \& Albertó, 2002). Las especies que conforman el género son saprobiontes, encontrándose en el suelo, pastizales, bosques 
Revisión del género Macrolepiota Singer (Agaricales, Basidiomycota) en Chile - Salazar-Vidal V. et al

deciduos, de coníferas, plantaciones, al borde de caminos, y zonas ricas en nutrientes, creciendo generalmente de modo gregario (Vellinga, 2001).

Se caracteriza por poseer basidiomas grandes, carnosos, y generalmente con escamas sobre el píleo; el himenio está formado por lamelas de color blanco a crema, siendo en algunas especies rosadas en la madurez; con un estípite bulboso en la base, un anillo prominente que se encuentra mayormente presente y, que en algunos casos es removible. Posee esporas de gran tamaño generalmente con una longitud mayor a $10 \mu \mathrm{m}$ (Vellinga, 2003), con paredes gruesas, donde se puede observar una reacción metacromática en su pared interna utilizando azul de cresilo, y la presencia de hifas fibuladas (Singer, 1948).

Vellinga \& De Kok (2002) propusieron que algunas especies del género Macrolepiota sean transferidas al género Chlorophyllum Massee, debido a la presencia de una himenodermis en el píleo en lugar de una tricodermis y, de un poro germinativo generado por una depresión en el episporio, en lugar de ser producido por una interrupción en éste y cubierto por una capa hialina como ocurre en Macrolepiota.

En Chile se han registrado tres especies del género Macrolepiota: M. procera (Scop.) Singer, M. bonaerensis (Speg.) Singer y M. rachodes (Vittad.) Singer, siendo esta última transferida al género Chlorophyllum. Estas tres especies han sido descritas como comestibles, sin embargo, pueden ser confundidas con algunas especies tóxicas del género Lepiota y Chlorophyllum.

\section{Clave dicotómica}

1. Píleo de $4-10 \mathrm{~cm}$ de diámetro, estípite de 7,2 - 26 x 0,7 - 1,1 cm. Presenta queilocistidios y pleurocistidios capitados

M. bonaerensis
1'. Píleo superior a $7 \mathrm{~cm}$ de diámetro. Ausencia de pleurocistidios.

2. Píleo entre 7 - $30 \mathrm{~cm}$ de diámetro, estípite de 10 - 34 x 1 - 2,2 cm adelgazándose hacia el ápice. Queilocistidios utriformes, claviformes

M. procera

2'. Píleo entre $9-18 \mathrm{~cm}$ de diámetro, estípite de 10 - 20 x 1,2 - $3 \mathrm{~cm}$. Queilocistidios vesiculosos, claviformes, de 18 - $27 \times 10-15 \mu \mathrm{m}$.

C. rachodes

\section{Macrolepiota procera (Scop.) Singer}

Características: Basidioma de mediano a gran tamaño. Píleo umbonado entre 7 - $30 \mathrm{~cm}$ de diámetro, ovoide cuando joven y aplanado-convexo al madurar, de color blanco a crema, con restos de velo universal en forma de escamas pardas, pardas oscuras a grisáceas. Láminas libres, densas, delgadas, blancas cuando joven, y crémeas al madurar. Estípite blanco, con unas bandas irregulares de colores pardos, cilíndrico de 10 - 34 x 1-2,2 cm adelgazándose hacia el ápice, y en la base posee un bulbo notorio. Anillo membranoso, ubicado en la zona superior, de color blanco, con la parte inferior parda. Esporas elípticas a ovoides, de 12 - 19 x $8-12 \mu \mathrm{m}$, de paredes gruesas, lisas, hialinas, dextrinoides, metacromáticas, con poro germinativo. Basidios claviformes de $30-43$ x 12 - 17 $\mu \mathrm{m}$, de paredes delgadas, hialinos, tetraesporados. Queilocistidios utriformes, claviformes a irregularmente claviformes, de 20 - 39 x 10 - $23 \mu \mathrm{m}$, de paredes delgadas, hialinos, en racimos, formando un borde estéril. Pleurocistidios ausentes (Ge et al., 2010).

Hábitat y ecología: Crece en el suelo, principalmente, fructifica en claros de bosques, pastizales, bordes de camino y praderas con Coigüe (N. dombeyi) y Hualle (N. obliqua), solitaria o en grupos, es una especie saprófita. En Chile se distribuye 
desde Viña del Mar hasta Valdivia. Los carpóforos se pueden recolectar desde mayo hasta principios de junio (Valenzuela, 2003).

Comentarios: Posee olor y sabor a nuez. M. procera es una especie comestible, siendo el píleo el que posee valor gastronómico. Sin embargo, puede ser confundida con $C$. rachodes, y otras especies que no se encuentran en Chile, como $M$. fuligineosquarrosa, M. fuliginosa, y M. permixta (Valenzuela, 2003).

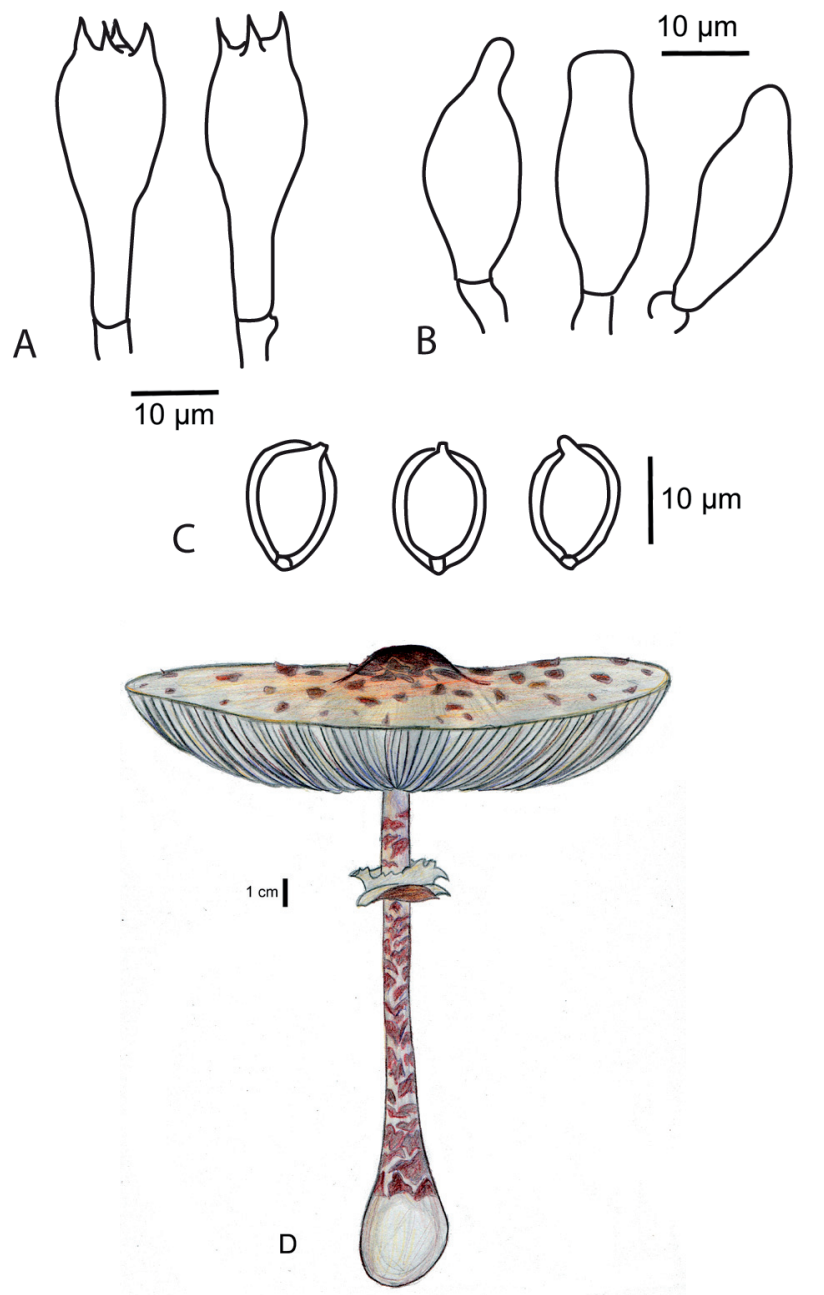

Figura 1. Macrolepiota procera. A) basidios, B) queilocistidios, y C) esporas. Ilustraciones adaptadas de Ge et al. (2010), D) basidioma.

\section{Macrolepiota bonaerensis (Speg.) Singer}

Características: Píleo umbonado de $4-10 \mathrm{~cm}$ de diámetro, ovoide a aplanado - convexo cuando maduro, de color blanco a crema, con restos de velo universal en forma de escamas marrón a marrón-rojizo, con el centro rojizo-castaño. Láminas libres, anchas, blancas cuando joven, y rosa al madurar. Estípite pardo blanquecino, con finas escámulas, cilíndrico de 7,2 - 26 x 0,7 - 1,1 cm, bulboso. Anillo membranoso, ubicado en la zona superior, de color blanco, con doble margen, con escamas pardo rojizas por debajo. Esporas elípticas a ovoides, de 12 - 19 × 9 - $13 \mu \mathrm{m}$, lisas, hialinas, dextrinoides, con poro germinativo. Basidios claviformes, tetrasporados, de $26-30 \times 8-11 \mu \mathrm{m}$ (Wright \& Albertó, 2002). Queilo y Pleurocistidios capitados, hialinos, de 41-75 x 12-15 $\mu \mathrm{m}$ (Garrido, 1988; Lazo, 2016). Sin embargo, Wright \& Albertó (2002) no detectaron la presencia de pleurocistidios.

Hábitat y ecología: Crece en el suelo, al borde de caminos, en praderas entre el pasto, fuera de zonas cubiertas por árboles (Lazo, 2016). Garrido (1988) la documentó bajo Peumo (Cryptocarya alba), Olivillo (Aextoxicon punctatum), Nirre (Nothofagus antarctica), Hualle (N. obliqua), Eucaliptus (Eucalyptus globosus), y Pino (Pinus radiata). En Chile se ha documentado en la zona central y austral (Lazo, 2016). Fructifica desde finales de abril hasta principios de mayo (Valenzuela, 2003).

Comentarios: Según Garrido (1988), posee aroma a miel o maní, y sabor similar al espárrago, mientras que Lazo (2016) describe su sabor como farináceo. $M$. bonaerensis es muy parecida a $M$. procera, sin embargo, es de color más claro, y escamas más pequeñas (Lazo, 2016). Wright \& Albertó (2002) la describen como una especie comestible, sin embargo, Lazo (2016) no recomienda su consumo. 

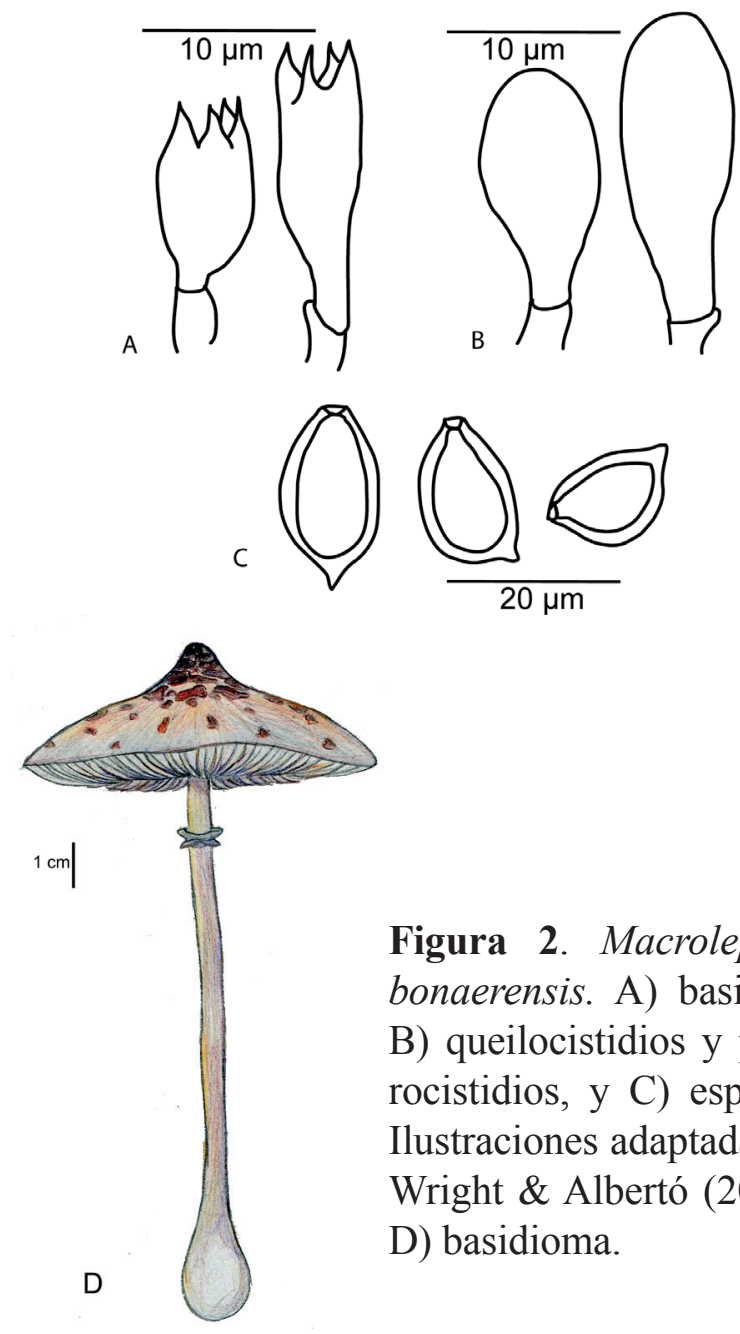

Figura 2. Macrolepiota bonaerensis. A) basidios, B) queilocistidios y pleurocistidios, y C) esporas. Ilustraciones adaptadas de Wright \& Albertó (2002), D) basidioma.

\section{Chlorophyllum rachodes (Vittad.) Vellinga}

Características: Píleo ligeramente umbonado, de 9 - $18 \mathrm{~cm}$ de diámetro, convexo a aplanado, de color blanco a crema, con restos de velo universal en forma de escamas de color canela grisáceo. Láminas libres, anchas, juntas, blancas cuando joven, $y$ amarillento al madurar. Estípite blanquecino, glabro, cilíndrico de $10-20 \times 1,2-3 \mathrm{~cm}$, bulboso. Anillo grueso, doble, membranoso, blanquecino. Esporas sub-elipsoidales, de 8 - 10,5 x 5 - $6 \mu \mathrm{m}$, lisas, hialinas, dextrinoides, metacromáticas, con poro germinativo. Basidios claviformes, tetrasporados de 22 - 34 x 7 - $9 \mu \mathrm{m}$. Queilocistidios vesiculosos, claviformes, de 18 - 27 x 10 - $15 \mu \mathrm{m}$. Pleurocistidios ausentes (Lazo, 2016).
Hábitat y ecología: Crece en el suelo, en lugares pantanosos, dentro de bosques y arboledas. En Chile se distribuye ampliamente en la zona central y austral (Lazo, 2016). Fructifica desde mayo hasta principios de junio (Valenzuela, 2003).

Comentarios: Posee un sabor discreto, aroma suave y consistencia fibrosa. Es una especie comestible, que se torna de un color pardo rojizo al romperse (Lazo, 2016), lo que en ciertas especies significa toxicidad, pero en este caso corresponde a una excepción.
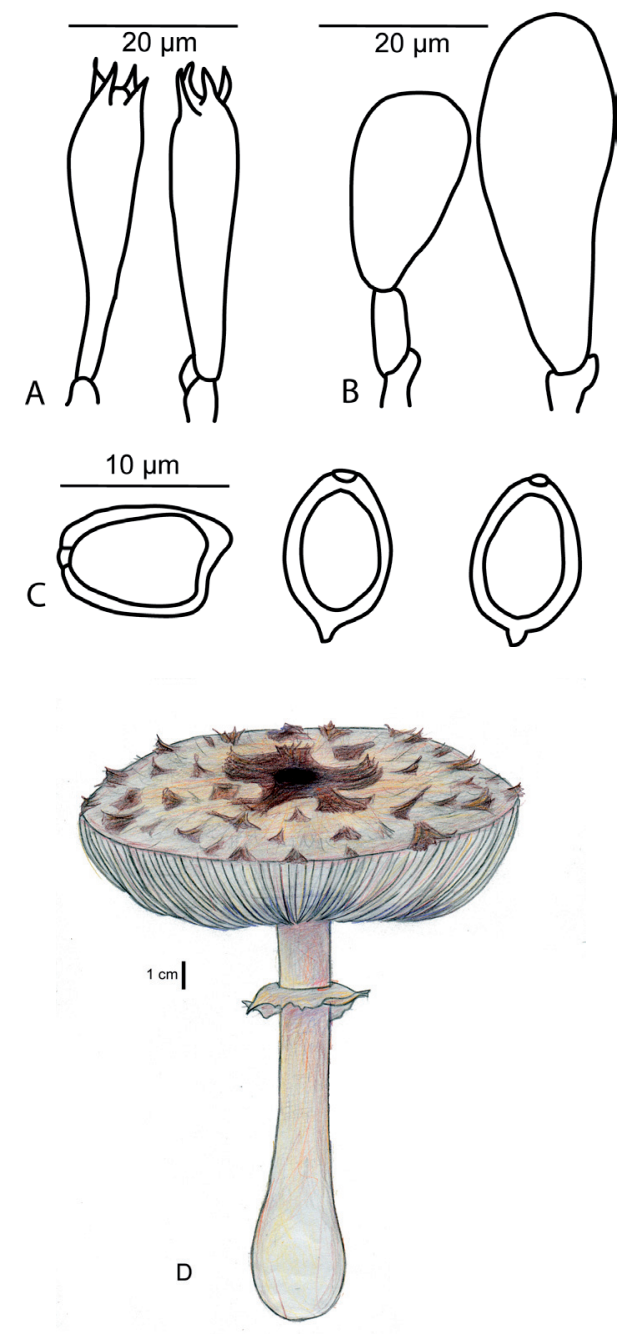

Figura 3. Chlorophyllum rachodes. A) basidios, B) queilocistidios, y C) esporas. Ilustraciones adaptadas de Wright \& Albertó (2002), D) basidioma. 
Revisión del género Macrolepiota Singer (Agaricales, Basidiomycota) en Chile - Salazar-Vidal V. et al

\section{Observaciones:}

Si bien, Kirk et al. (2008) señalan que existen 30 especies del género Macrolepiota distribuidas a nivel mundial, Roskov et al. (2017) documentan a través de un catálogo online actualizado de especies registradas hasta ahora, que este género cuenta con 42 especies.

En Chile, aún se continúa utilizando $M$. rachodes como sinónimo de $C$. rachodes, por lo cual en esta revisión los autores decidieron incluir esta especie.

Es necesario señalar que de las tres especies presentes en Chile, $C$. rachodes es la que posee las escamas más prominentes y el estípite más grueso. Por su parte, M. procera posee unas franjas atigradas notorias en el estípite, siendo en algunos casos distribuidas a lo largo de éste y en otros sólo en la parte cercana a la base.

Finalmente, mencionar que los autores no se hacen responsables de una determinación equivocada de las especies mencionadas en esta revisión, ni de posibles intoxicaciones debido a su consumo, puesto que las especies del género Macrolepiota pueden ser confundidas fácilmente en su estado juvenil con algunas especies tóxicas del género Lepiota y otros géneros. Además, Lazo (2016) insiste en aconsejar no comer jamás setas crudas, por comestibles que ellas sean cuando estén cocinadas, pues algunas contienen cancerígenos u otras sustancias que sólo se destruyen mediante la cocción.

\section{AGRADECIMIENTOS}

Los autores agradecen al Dr. Edgardo A1bertó, profesor adjunto de la Universidad Nacional de General San Martín y al Dr. Zu Yang, investigador de Kunming Institute of Botany of the Chinese Academy of Sciences, por permitirnos utilizar adaptaciones de los dibujos de las esporas, cistidios y basidios correspondientes a cada especie. A nuestro colega Julio Becerra, por facilitarnos literatura pertinente para esta revisión.

\section{REFERENCIAS}

Garrido, N. (1988). Agaricales s.l. und ihre Mykorrizen in den Nothofagus-Wäldern Mittelchiles (Agaricales s.l. y sus micorrizas en los bosques de Nothofagus en Chile central). Bibliotheca Mycologica 120, J. Cramer, Berlin, Stuttgart, ISBN 3-44359021-7.

Ge, Z. W., Yang, Z. L., \& Vellinga, E. C. (2010). The genus Macrolepiota (Agaricaceae, Basidiomycota) in China. Fungal Diversity, 45(1), 81-98.

Global Biodiversity Information Facility (GBIF). [En línea] http://www.gbif.org/species/2536706. Última consulta: 15/06/2017

Kirk, P. M., Cannon, P. F., Minter, D. W., \& Stalpers, J. A. (2008). Dictionary of the Fungi. 10th edition. Wallingford, UK.
Lazo, W. (2016). Hongos de Chile Atlas Micológico. Segunda edición. Facultad de Ciencias de la Universidad de Chile. Editorial Universitaria, Chile. 316 pp.

Roskov, Y., Abucay, L., Orrell, T., Nicolson, D., Bailly, N., Kirk, P.M., et al., eds. (2017). Species 2000 \& ITIS Catalogue of Life, 2017 Annual Checklist. Digital resource at www.catalogueoflife.org/annual-checklist/2017. Species 2000: Naturalis, Leiden, the Netherlands. ISSN 2405-884X.

Singer, R. (1948). New and interesting species of Basidiomycetes. II. Pap. Papers Michigan Academy of Science, Arts and Letters, 32, 103-150.

Valenzuela, E. (2003). Hongos comestibles silvestres colectados en la X región de Chile. Boletín Micológico, 18. 
Vellinga, E. C. (2001). Macrolepiota Sing. Flora Agaricina Neerlandica. Critical monographs on families of agarics and boleti occurring in the Netherlands, 5, 64-73.

Vellinga, E. C. (2003). Chlorophyllum and Macrolepiota (Agaricaceae) in Australia. Australian Systematic Botany, 16(3), 361-370.
Vellinga, E. C., de Kok, R. P., \& Bruns, T. D. (2003). Phylogeny and taxonomy of Macrolepiota (Agaricaceae). Mycologia, 95 (3), 442-456.

Wright, J. E., \& Albertó, E. (2002). Guia de los Hongos de la región Pampeana: Hongos con Laminillas. Editorial L.O.L.A. Buenos Aires. 